\title{
Naked strong curvature singularities in Szekeres space-times
}

\author{
Pankaj S. Joshi \\ Theoretical Astrophysics Group, \\ Tata Institute of Fundamental Research, \\ Homi Bhabha Road, Bombay 400 005, India \\ Andrzej Królak \\ Institute of Mathematics, Polish Academy of Sciences, \\ Śniadeckich 8, 00-950 Warsaw, Poland.
}

\begin{abstract}
We investigate the occurrence and nature of naked singularities in the Szekeres space-times. These space-times represent irrotational dust. They do not have any Killing vectors and they are generalisations of the Tolman-Bondi-Lemaitre space-times. It is shown that in these space-times there exist naked singularities that satisfy both the limiting focusing condition and the strong limiting focusing condition. The implications of this result for the cosmic censorship hypothesis are discussed.
\end{abstract}


There exist many explicit solutions of the Einstein equations that exhibit naked singularities. Nevertheless the cosmic censorship hypothesis can still be true if these singularities could be shown to be in some sense non-generic. It was supposed by Tipler [1] and independently by Królak [2] that all generic singularities are of strong curvature type. We say that a future-incomplete (respectively past-incomplete) causal geodesic terminates in a strong curvature singularity in the future (respectively in the past) if for every point $q \in \lambda$ the expansion $\theta$ of the congruence of the future-directed (respectively pastdirected) causal geodesics originating from $\mathrm{q}$ and infinitesimally neighbouring $\lambda$ diverges (Tipler's strong curvature singularity) (becomes negative (Królak's strong curvature singularity)). We say that strong curvature condition holds if all future and past incomplete null geodesics generating an achronal set terminate in a strong curvature singularity. It was conjectured by Królak [3] that under strong curvature condition cosmic censorship holds. Subsequent work has shown that Królak's conjecture is not true. On the one hand the censorship theorems proved by Królak [4 and later by Beem and Królak [5] needed an extra restrictive assumption on causal structure of space-times and on the other hand explicit examples of naked strong curvature singularities in Królak's sense ( 6, 7, 8]) and in Tipler's sense (19, 10, 11]) were demonstrated in the Tolman-Bondi-Lemaître (TBL) space-times representing sphericaly symmetric inhomogeneous collapse of dust and in Vaidya radiation collapse. The TBL space-times are special in two senses: they are spherically symmetric and they have matter in the form of irrotational pressureless dust. It is interesting to know whether naked strong curvature singularities occur in more general space-times. In this letter we show that naked strong curvature singularities occur in Szekers space-time that do not have any Killing vector. This result shows that naked strong curvature singularites do not arise as a result of spherical symmetry. Nevertheless the Szekeres space-times have the same special form of matter as TBL space-times i.e. irrotational pressureless dust.

The Szekeres space-time [12 is a solution of Einstein's equations representing irrotational dust

$$
G_{a b}=T_{a b}=\rho u_{a} u_{b}, \quad u_{a} u^{a}=1,
$$

where units are chosen so that $c=8 \pi G=1$. The metric has the diagonal form

$$
d s^{2}=d t^{2}-X^{2} d r^{2}-Y^{2}\left(d x^{2}+d y^{2}\right),
$$


where $(r, x, y)$ are comoving spatial coordinates. The solution is given by (we consider the case $Y^{\prime}=\frac{\partial Y}{\partial r} \neq 0$ )

$$
Y=\frac{R(t, r)}{P(r, x, y)}, \quad X=\frac{P(r, x, y) Y^{\prime}(t, r)}{\sqrt{1+f(r)}},
$$

where $f(r)>-1$ and

$$
\begin{aligned}
P= & a(r)\left(x^{2}+y^{2}\right)+2 b_{1}(r) x+2 b_{2}(r) y+c(r), \\
& a c-b_{1}^{2}-b_{2}^{2}=\frac{1}{4} \\
& \dot{R}^{2}=f+\frac{F(r)}{R}
\end{aligned}
$$

where $F(r)$ is an arbitrary function of $r$ and where dot denotes partial derivative w.r.t time coordinate $t$.

We assume the following regularity conditions.

1. The metric is everywhere $C^{1}$. Then the function $P$ must be everywhere non-zero and its derivative w.r.t $r$ must be continuous and vanishing at $r=0$.

2. The metric is locally Euclidean at $r=0$. Then it is necessary to set

$$
f(0)=0 .
$$

3. The function $R_{o}(r)=R(r, 0)$ is a monotonically increasing function of $r$. We can then use the freedom in the choice of the radial coordinate $r$ to obtain

$$
R_{o}(r)=r
$$

The dust density $\rho$ is given by

$$
\rho=\frac{P F^{\prime}-3 F P^{\prime}}{P^{2} R^{2} Y^{\prime}} .
$$

Although for $P>0$ the surfaces $r=$ const, $t=$ const are spheres, the solution is not spherically symmetric because the spheres are not concentric, their centers are given by $\left(-a^{-1} b_{1},-a^{-1} b_{2}\right)$. Szekeres has also analysed the singularities and their causal structure in his space-times. When $R=0$, the singularity is of the first kind, and when $Y^{\prime}=0$ the singularity is of the second kind. The singularities of the second kind are familiar shell-crossing 
singularities that also occur in TBL space-times [13]. Like in the TBL spacetimes shell-crossing singularities in Szekeres spaces can also be both locally and globally naked [12]. However they are generally believed to be mild and we shall not consider them here. We shall eliminate these singularies by imposing a regularity condition

$$
Y^{\prime}>0
$$

Szekeres has also shown that whenever $r>0$ the shell of dust always crosses the apparent horizon before collapsing to singularity and therefore for $r>0$ the singularity cannot be naked. Therefore the singularity of the first kind can be naked only when $r=0$ which we call the central singularity. This situation is analogous to the TBL case. We shall show that like in TBL space-times naked strong curvature singualrities do occur in Szekeres spacetimes. We shall consider the case of gravitational collapse i.e. we shall require $\dot{R}<0$. For simplicity we shall only consider the case of mariginally bound collapse i.e. we set

$$
f(r)=0 .
$$

Then the function $R(r, t)$ is given by

$$
R=r\left(1-\frac{3}{2} \sqrt{\frac{F}{r^{3}}} t\right)^{2 / 3} .
$$

Our analysis follows that of Joshi and Dwivedi for the TBL case [9]. We introduce a set of new functions:

$$
\begin{aligned}
X & =\frac{R}{r^{\alpha}}, \\
\eta & =r \frac{F^{\prime}}{F}, \\
\Lambda & =\frac{F}{r^{\alpha}}, \\
\Theta & =\frac{1-\frac{1}{3} \eta}{r^{\frac{3(\alpha-1)}{2}}}, \\
\mathcal{L} & =r \frac{P^{\prime}}{P},
\end{aligned}
$$

where $\alpha \geq 1$ and the unique value of the constant $\alpha$ is determined by the condition that $\frac{\Theta}{\sqrt{X}}$ does not vanish or goes to infinity identically as $r \rightarrow 0$ 
in the limit of approach to the central singularity along any $X=$ const direction. We shall assume that the above functions are at least $C^{2}$. Partial derivatives $R^{\prime}$ and $\dot{R}^{\prime}$ that are important in the analysis of the singularity are then given by

$$
\begin{aligned}
\dot{R} & =-\sqrt{\frac{\Lambda}{X}}, \\
R^{\prime} & =r^{\alpha-1} H, \\
\dot{R}^{\prime} & =-\frac{N}{r},
\end{aligned}
$$

where

$$
\begin{aligned}
H & =\frac{1}{3} \eta X+\frac{\Theta}{\sqrt{X}} \\
N & =-\frac{\sqrt{\Lambda}}{2 X^{2}}\left(\Theta-\frac{2}{3} \eta X^{3 / 2}\right) .
\end{aligned}
$$

The tangents $K^{a}=d x^{a} / d k$ for the outgoing radial $(x=$ const, $y=$ const $)$ null geodesics can be written as

$$
\begin{aligned}
K^{t} & =\frac{d t}{d k}=\frac{\mathcal{P}}{Y}, \\
K^{r} & =\frac{d r}{d k}=\frac{\mathcal{P}}{P Y Y^{\prime}}, \\
K^{x} & =\frac{d x}{d t}=0, \\
K^{y} & =\frac{d y}{d t}=0,
\end{aligned}
$$

where $\mathcal{P}$ satisfies the differential equation

$$
\frac{d \mathcal{P}}{d k}+\mathcal{P}^{2}\left(\frac{\dot{Y}^{\prime}}{Y Y^{\prime}}-\frac{\dot{Y}}{Y^{2}}-\frac{1}{P Y^{2}}\right)=0
$$

The parameter $k$ is an affine parameter along the null geodesics.

If the outgoing null geodesics are to terminate in the past at the central singularity $r=0$, which occurs at some time $t=t_{o}$ at which $R\left(t_{o}, 0\right)=0$, 
then along such geodesics we have $R \rightarrow 0$ as $r \rightarrow 0$. The following is satisfied along null geodesics

$$
\begin{aligned}
\frac{d R}{d u}= & \frac{1}{\alpha r^{\alpha-1}}\left[\dot{R} \frac{d t}{d r}+R^{\prime}\right]= \\
& \left(1-\sqrt{\frac{\Lambda}{X}}\right) \frac{H(X, u)}{\alpha}-\frac{\sqrt{X \Lambda}}{\alpha} \mathcal{L} \equiv U(X, u),
\end{aligned}
$$

where we have put $u=r^{\alpha}$. Let us consider the limit $X_{o}$ of the function $X$ along the null geodesic terminating at the sigularity at $R=0, u=0$. Using the l'Hospital rule we get

$$
X_{o}=\lim _{R \rightarrow 0, u \rightarrow 0} \frac{R}{u}=\lim _{R \rightarrow 0, u \rightarrow 0} \frac{d R}{d u}=\lim _{R \rightarrow 0, u \rightarrow 0} U(X, u)=U\left(X_{o}, 0\right)
$$

The necessary condition for the existence of the null geodesic outgoing from the central singularity is the existence of the positive real root $X_{o}$ of the equation

$$
V(X) \equiv U(X, 0)-X=0 .
$$

By our regularity conditions we have that $\lim _{r \rightarrow 0} \mathcal{L}=0$. Consequently the necessary condition for the existence of the naked singularity in the mariginally bound case of Szekeres space-time is the existence of positive real root of the equation

$$
\left(1+\sqrt{\frac{\Lambda_{o}}{X}}\right) \frac{H(X, 0)}{\alpha}-X=0
$$

where we put

$$
\begin{gathered}
\eta_{o}=\eta(0), \\
\Lambda_{o}=\Lambda(0), \\
\Theta_{o}=\Theta(0) .
\end{gathered}
$$

This is exactly the same equation as in the mariginally bound TBL case [9]. Consequently the same analysis as in the TBL case applies here. We shall only summarize here a few key results. To show that the singularity is naked we still need to prove that there exists a solution of the geodesic equation such that the tangent $X_{o}$ is realized at the singularity. One can 
prove that there is always at least a single null geodesic outgoing from the central singularity ([9] p.5363). Thus the existence of the real and positive root of the equation (39) is both necessary and sufficient condition for the existence of a naked singularity.

We shall next investigate the strength of the singularity. Let $M$ denote the space-time manifold. Let $J(k)$ be defined on a null geodesic $\lambda:\left(k_{o}, 0\right] \rightarrow M$ parameterised by an affine parameter $k$

$$
J(k)=\int_{k_{o}}^{0} R_{a b} K^{a} K^{b} d k^{\prime}
$$

We say that the limiting focusing condition (LFC) holds if $J(k)$ is unbounded in the interval $\left(k_{o}, 0\right]$ and we say that the strong limiting focusing condition (strong LFC) if $J(k)$ is non-integrable on an interval $\left(k_{o}, 0\right]$. It is proved in Ref. 14 that LFC implies that $\lambda$ terminates in Królak's strong curvature singularity in the future whereas strong LFC implies that $\lambda$ terminates in Tipler's strong curvature singularity in the future.

To find out whether the naked singularity satisfies strong limiting focusing condition we shall investigate the $\operatorname{limit}_{\lim _{k \rightarrow 0}} k^{2} R_{a b} K^{a} K^{b}$ along the future directed null geodesics coming out from the singularity. Using the l'Hospital rule, regularity conditions and Eq.27 we have

$$
\lim _{k \rightarrow 0} k^{2} R_{a b} K^{a} K^{b}=\lim _{k \rightarrow 0} \frac{k^{2}\left(F^{\prime}-3 F \frac{P^{\prime}}{P}\right)\left(K^{t}\right)^{2}}{R^{2}\left(R^{\prime}-R \frac{P^{\prime}}{P}\right)}=\frac{\eta_{o} \Lambda_{o} H_{o}}{X_{o}^{2}\left(\alpha-N_{o}\right)^{2}},
$$

where $N_{o}=N\left(X_{o}, 0\right)$. Thus if $\Lambda_{o} \neq 0$ the naked singularity satisfies the strong limiting focusing condition.

Suppose then that $\Lambda_{o}=0$. We can write the Eq.27 in the following form

$$
\frac{d\left(\ln K^{t}\right)}{d k}=\left(N-\sqrt{\frac{\Lambda}{X}} \mathcal{L}\right) \frac{1}{r} \frac{d r}{d k} .
$$

Since as $k \rightarrow 0, N \rightarrow 0$ because $\Lambda_{o}=0$ and $\mathcal{L} \rightarrow 0$ by regularity condition the right hand side of the above equation is integrable on the interval $\left(k_{o}, 0\right]$

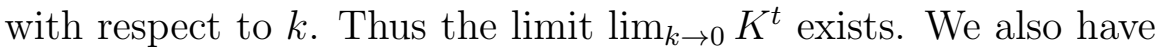

$$
K^{r}=\frac{K^{t}}{r^{\alpha-1}(H-X \mathcal{L})} .
$$


Thus if $\alpha=1$ the $\operatorname{limit}_{k \rightarrow 0} \lim ^{r}$ also exists. Let us suppose thet $\alpha=1$ and let us consider the $\operatorname{limit}_{\lim _{k \rightarrow 0}} k R_{a b} K^{a} K^{b}$ Applying the l'Hospital rule twice and using Eq.27 we get

$$
\lim _{k \rightarrow 0} k R_{a b} K^{a} K^{b}=\frac{\eta_{o} \Lambda_{o}^{\prime} H_{o}}{X_{o}^{2}\left(\alpha-N_{o}\right)^{2}} \lim _{k \rightarrow 0} K^{r} .
$$

Thus when $\alpha=1$ the above limit is finite and the naked singularity just satisfies the limiting focusing condition $\left(R_{a b} K^{a} K^{b}\right.$ diverges logarithmically). If $\alpha>1$ the above limit diverges and the naked singularity also satisfies the limiting focusing condition (though not strong focusing condition unless $\left.\Lambda_{o} \neq 0\right)$. The above results show that under the regularity conditions given above and for mariginally bound collapse any central naked singularity in

the Szekeres space-time is always a strong curvature singularity in Królak's sense.

\section{Acknowledgements}

This work was supported in part by Polish Science Council grant KBN 2 P301 05007.

\section{References}

[1] Tipler F J 1977 Phys. Lett.67A 8

[2] Królak A 1978 MSc Thesis, Singularities and Black Holes in General Space-times University of Warsaw, unpublished

[3] Królak A 1983 Gen. Rel. Grav. 1599

[4] Królak A 1986 Class. Quantum Grav.3 267

[5] Beem J K and Królak A J. Math. Phys. 332249

[6] Eardley D M and Smarr L 1979 Phys. Rev. D 192239

[7] Christodoulou D 1984 Commun. Math. Phys.93 171

[8] Newman R P A C 1986 Class. Quantum Grav.3 527 
[9] Joshi P S and Dwivedi I H 1993 Phys. Rev. D 475357

[10] Dwivedi I H and Joshi P S 1989 Class. Quantum Grav.6 1599

[11] Dwivedi I H and Joshi P S 1991 Class. Quantum Grav.8 1339

[12] Szekeres P 1975 Phys. Rev.D12 2941

[13] Yodzis P Seifert H J and Muller zum Hagen 1973 Commun. Math. Phys.34 135

[14] Clarke C J S and Królak A 1985 J. Geom. Phys.2 127 